\title{
Re-irradiation of unresectable recurrent head and neck cancer: using Helical Tomotherapy as image-guided intensity-modulated radiotherapy
}

\author{
Songmi Jeong, $M D^{1}$, Eun Jung Yoo, MD', Ji Yoon Kim, MD', \\ Chi Wha Han, MD², Ki Jun Kim, MD³ ${ }^{3}$ Chul Seung Kay, MD \\ Departments of ${ }^{1}$ Radiation Oncology, ${ }^{2}$ Internal Medicine, and ${ }^{3}$ Diagnostic Radiology, \\ The Catholic University of Korea College of Medicine, Seoul, Korea
}

Purpose: Re-irradiation (re-RT) is considered a treatment option for inoperable locoregionally recurrent head and neck cancer (HNC) after prior radiotherapy. We evaluated the efficacy and safety of re-RT using Helical Tomotherapy as image-guided intensitymodulated radiotherapy in recurrent HNC.

Materials and Methods: Patients diagnosed with recurrent HNC and received re-RT were retrospectively reviewed. Primary endpoint was overall survival (OS) and secondary endpoints were locoregional control and toxicities.

Results: The median follow-up period of total 9 patients was 18.7 months (range, 4.1 to 76 months) and that of 3 alive patients was 49 months (range, 47 to 76 months). Median dose of first radiotherapy and re-RT was 64.8 and $47.5 \mathrm{~Gy}{ }_{10}$. Median cumulative dose of the two courses of radiotherapy was $116.3 \mathrm{~Gy}_{10}$ (range, 91.8 to $128.9 \mathrm{~Gy}_{10}$ ) while the median interval between the two courses of radiation was 25 months (range, 4 to 137 months). The response rate after re-RT of the evaluated 8 patients was $75 \%$ (complete response, 4; partial response, 2). Median locoregional relapse-free survival after re-RT was 11.9 months (range, 3.4 to 75.1 months) and 5 patients eventually presented with treatment failure (in-field failure, 2; in- and out-field failure, 2; out-field failure, 1). Median OS of the 8 patients was 20.3 months (range, 4.1 to 75.1 months). One- and two-year OS rates were 62.5\% and $50 \%$, respectively. Grade 3 leucopenia developed in one patient as acute toxicity, and grade 2 osteonecrosis and trismus as chronic toxicity in another patient.

Conclusion: Re-RT using Helical Tomotherapy for previously irradiated patients with unresectable locoregionally recurrent HNC may be a feasible treatment option with long-term survival and acceptable toxicities.

Keywords: Recurrent head and neck cancer, Re-RT, Helical Tomotherapy, IG-IMRT, Intensity-modulated radiotherapy, Image-guided radiation therapy

\section{Introduction}

The management of recurrent head and neck cancer (HNC) remains a challenge despite evolutionary therapeutic options and treatment intensification. About 30\% to 50\% of patients with locally advanced tumor recurred locoregionally $[1,2]$, and some investigators reported that more than half of treated patients developed locoregional recurrence [3-5]. The

Received 17 September 2013, Revised 15 October 2013, Accepted 24 October 2013.

Correspondence: Chul Seung Kay, MD, Department of Radiation Oncology, Incheon St. Mary's Hospital, The Catholic University of Korea College of Medicine, 56 Dongsu-ro, Bupyeong-gu, Incheon 403-720, Korea. Tel: +82-32-280-6026, Fax: +82-32-280-6024, E-mail: kaycs@catholic.ac.kr

(c) This is an Open Access article distributed under the terms of the Creative Commons Attribution Non-Commercial License (http://creativecommons.org/ licenses/by-nc/3.0/) which permits unrestricted non-commercial use, distribution, and reproduction in any medium, provided the original work is properly cited.

www.e-roj.org 
treatment of choice for previously irradiated recurrent HNC is salvage surgery. However, many patients are in inoperable state or it is difficult to achieve complete resection with no residual disease because recurrent tumors often infiltrate into adjacent structures or extensive fibrosis caused by prior treatment complicates surgical procedures. For those who cannot undergo surgery, systemic chemotherapy was the most frequently considered treatment option but nevertheless, relatively low response rates of 20\% to 35\% were reported [6]. Thus the role of chemotherapy was limited to palliative aim with median survival of 5 to 6 months and survival was not elongated satisfactorily long enough $[7,8]$.

Radiotherapy (RT) may be an option in treating recurrent $\mathrm{HNC}$, but re-irradiation to the previously irradiated head and neck area has its limitations caused by prior dose irradiated to normal tissues. This makes it difficult to prescribe a curative radiation dose to the recurrent lesion. Several groups reported that re-irradiation could achieve long-term survival in selected recurrent HNC patients, but may accompany substantially severe complications [9-11]. However, recent improvement of RT techniques and dose escalation using intensity-modulated radiotherapy (IMRT) have generated relatively higher curative dose delivery to the gross target while sparing more normal tissues in overlapping head and neck fields. Phase II study of the Radiation Therapy Oncology Group (RTOG) and other single-institutions documented that re-irradiation using IMRT technique was tolerable and feasible [12-14]. Furthermore, the use of image-guided radiation therapy (IGRT) contributes in reducing set up uncertainties $[15,16]$. The purpose of this study was to investigate the efficacy and safety of re-irradiation in locoregionally recurrent HNC using Helical Tomotherapy as IGIMRT.

\section{Materials and Methods}

\section{Patient selection}

From February 2006 to September 2009, a total of 9 patients with locoregionally recurrent $\mathrm{HNC}$ who received re-irradiation as IG-IMRT using Helical Tomotherapy to the head and neck areas were retrospectively reviewed. Recurrence was defined as development of new lesion or disease progression after the prior treatment. All patients were initially locally advanced HNC (stage III or IVa) and had received external beam RT with combined surgery or chemotherapy if clinically indicated. Histological diagnosis or clinical evaluation of recurrence was completed before the start of re-irradiation, including tissue biopsy, physical examination, laryngoscopy, computed tomography (CT), magnetic resonance imaging (MRI) and/ or positron emission tomography-CT (PET-CT). Patients with synchronous distant metastases at the time of re-irradiation were excluded from this analysis (2 patients).

\section{Treatment}

All patients were re-irradiated with Hi-Art Tomotherapy system (TomoTherapy Inc., Madison, WI, USA), which is a helical fan-beam IMRT using 6-MV photon. The combination of multileaf collimator, field width and table speed generates a high degree of dose modulation and enhanced conformality. To reduce patient set up uncertainty, patients were fixed in supine position and were immobilized with thermoplastic head mask for optimal daily IGRT. Planning CT scans with 3-mm slice thickness were obtained in treatment position. Daily megavoltage CT (MVCT) images were obtained as a component of IGRT before irradiation. Obtained MVCTs were fused with the planning CT images and were adjusted by moving the patient couch if needed. A radiation oncologist reviewed and checked the fused CT images every day.

The recurrent tumor masses or metastatic lymph nodes were defined as gross tumor volume (GTV). Approximately $1.0 \mathrm{~cm}$ margin from GTV for microscopic tumor spread was defined as clinical target volume (CTV) and the CTV was modified to reduce re-irradiation dose of adjacent organs at risk (OARs), such as spinal cord, salivary glands, mandible, optic nerve, and major vessels. The planning target volume (PTV) was outlined considering patient motion and daily setup error with 0.3 $\mathrm{cm}$ expansion from the CTV. Elective nodal irradiation was given when regional lymph node metastasis was detected. Inverse treatment planning process with TomoTherapy $\mathrm{Hi}$ Art treatment planning system ver. 3.3.4 was used to deliver prescribed dose to the PTV while sparing critical structures. The planning goal was to ensure $>95 \%$ of PTV received 100\% of the prescribed dose without any PTV receiving $>115 \%$ and $<85 \%$ of the prescribed treatment dose. Another consideration was that not $>1 \%$ of the tissue outside the PTV received $>110 \%$ of the prescribed dose.

The PTV dose for re-irradiation was determined by neurologic OARs, such as spinal cord, brainstem or optic apparatus (optic nerves and chiasm). Attempts were made to deliver maximal cumulative dose of spinal cord, brainstem, and optic apparatus below $45 \mathrm{~Gy}, 54 \mathrm{~Gy}$, and $55 \mathrm{~Gy}$, respectively. If the treatment interval between initial RT and second RT was longer than 1 year, we assumed that the neurologic organs recovered the 
tolerance and allowed the cumulative dose to exceed the limits. Other non-neurologic OARs, such as salivary glands, mandible, glottic larynx, and esophagus were delineated and avoidance constraints were set at doses as low as possible without compromising the PTV dose coverage if they had been included in the prior RT field. If they were previously excluded from RT field and not highly irradiated, the mean dose constraints to the parotid glands were below $26 \mathrm{~Gy}$, the maximal dose constraints to the mandible were below $70 \mathrm{~Gy}$, and two-thirds of the glottic larynx were irradiated below $50 \mathrm{~Gy}$. Further attempts were made to minimize the volume of OARs and previously irradiated soft tissue outside PTV receiving $>60 \mathrm{~Gy}$.

The cumulative dose of radiation was calculated as the sum of prior RT dose and re-irradiation dose. Irradiated doses were converted into biologically effective doses (BED) using an $\alpha$ / $\beta$ ratio of 10 , based on the linear-quadratic model. Normalized iso-effective dose was calculated for fractional dose of 1.8 Gy using an estimated $\alpha / \beta$ ratio of 10 . No correction was made for variable times between the two courses of radiation therapy among patients. Time interval between the two RT courses was defined as from the last day of the prior RT to the first day of the 2 nd RT.

\section{Follow-up, response evaluation, and toxicity}

Follow-up period was defined as the day of recurrence to the date of expiration or the time of analysis, March 2013. Overall survival (OS) was defined as the first day of re-irradiation to the day of death. Locoregional relapse-free survival (LRRFS) was measured from the first day of re-irradiation to the date of relapse or expiration. Locoregional failure was defined as disease progression within the re-irradiation field. In case the patient is alive or there was no recurrence, LRRFS was determined by the time of the event documented at the time of analysis.

The response rate of re-irradiation was evaluated with imaging study and clinical examination using Response Evaluation Criteria in Solid Tumors after completion of the therapy. The first response evaluation after re-irradiation was performed with laryngoscopy, CT, MRI and/or PET-CT within 1 to 3 months and then follow-ups were performed every 3 months in the first year and every 6 to 12 months thereafter.

Treatment related acute and late toxicities were evaluated by physicians using Common Terminology Criteria for Adverse Events ver. 3.0. Acute toxicity was defined as a complication developed during the radiation treatment or within one to three months after re-irradiation completion. Late toxicity was defined as a complication developed thereafter.

\section{Statistical analysis}

The OS and LRRFS were analyzed using Kaplan-Meier method. Because of the small number of patients, comparison or defining factors which influenced survival could not be evaluated.

\section{Results}

\section{Patient characteristics \&t treatment}

Patient characteristics and details are described in Table 1. The median age of patients was 53 years (range, 32 to 82 years). Five patients were male and 4 patients were female. Among the total patients, squamous cell carcinoma histology was found in 8 patients (89\%). The majority of the patients, 6 out of 9 (67\%), were re-irradiated for local recurrence and the rest (3 patients, 33\%) were re-irradiated for both the local and regional recurrent lesions. Elective irradiation of adjacent

Table 1. Patient characteristics

\begin{tabular}{|c|c|c|c|c|c|c|c|}
\hline Patient no. & Age & Sex & Primary site & Initial stage & Histology & Recurred area & Recurrent stage \\
\hline 1 & 53 & $M$ & Right tongue & $\mathrm{T} 2 \mathrm{~N} 2 \mathrm{M} 0$ & $\mathrm{SqCC}$ & Locoregional & T4N1M0 \\
\hline 2 & 59 & M & Right glottis & T3NOMO & $\mathrm{SqCC}$ & Local & T4NOMO \\
\hline 3 & 70 & $\mathrm{~F}$ & Right nasopharynx & T1N2M0 & $\mathrm{SqCC}$ & Local & T4NOMO \\
\hline 4 & 45 & $\mathrm{M}$ & Right nasopharynx & T3N0MO & $\mathrm{SqCC}$ & Local & T4N0M0 \\
\hline 5 & 34 & $\mathrm{~F}$ & Right tongue & T3N2M0 & $\mathrm{SqCC}$ & Locoregional & T3N2Mo \\
\hline 6 & 62 & $M$ & Right soft palate & $\mathrm{T} 2 \mathrm{~N} 2 \mathrm{MO}$ & $\mathrm{SqCC}$ & Locoregional & T4N2M0 \\
\hline 7 & 82 & $\mathrm{~F}$ & Right hypopharynx & T4N0MO & $\mathrm{SqCC}$ & Local & T4N0M0 \\
\hline 8 & 42 & $\mathrm{~F}$ & Left maxillary sinus & T3NOMO & $\mathrm{SqCC}$ & Local & T4NOMO \\
\hline 9 & 32 & $M$ & Right maxillary sinus & T3N0M0 & $A C C$ & Local & T4NOMO \\
\hline
\end{tabular}

SqCC, squamous cell carcinoma; ACC, adenoid cystic carcinoma. 
uninvolved nodal regions was performed in the 3 patients with regional recurrence (patient no. 1,5, and 6)

Treatment characteristics and details are described in Table 2. Prior RT was performed using 2-demensional (2D) RT technique in 3 patients, 3-dimensional (3D) conformal RT in 5 patients and IMRT in 1 patient. The median dose of prior radiation was $64.8 \mathrm{~Gy}_{10}$, ranged from 50.4 to $73.8 \mathrm{~Gy}_{10}$, and the majority of the radiotherapies were given adjuvantly after tumor resection (6 patients). After the median time interval of 25 months (range, 4 to 137 months), re-irradiation was performed. Among all 9 patients, most patients received re-irradiation as RT alone (5 patients), 3 patients received weekly cisplatin-based concurrent chemoradiation and 1 patient received sequential RT after chemotherapy with taxol, cisplatin and 5-fluorouracil. The median re-irradiation dose to the recurrent lesion, converted into BED using an $\alpha / \beta$ ratio of 10 , was $47.5 \mathrm{~Gy}_{10}$ (range, 36.0 to $\left.67.1 \mathrm{~Gy}_{10}\right)$ and the median re-irradiation dose of the 8 patients who completed planned RT was $49.15 \mathrm{~Gy}_{10}$ with the median fraction size $2 \mathrm{~Gy}$ (range, 1.8 to $4 \mathrm{~Gy}$ ). One patient (patient no.
5) stopped RT at the dose of $38.7 \mathrm{~Gy}_{10}$ because the progression of the recurrent mass resulted in worsening of predisposed malignant trachea-esophageal fistula. Other patients were well compliant without treatment interruption. The absolute median PTV of re-irradiation was $107.71 \mathrm{~cm}^{3}$ (range, 14.58 to $340.09 \mathrm{~cm}^{3}$ ). The median cumulative dose of the two courses of RT was $116.3 \mathrm{~Gy}_{10}$ (range, 91.8 to $128.9 \mathrm{~Gy}_{10}$ ).

\section{Response and survival after the re-irradiation}

The median follow-up period was 18.7 months (range, 4.1 to 76 months) and the median follow-up period of the 3 live patients by the time of analysis was 49 months (range, 47 to 76 months). Treatment outcomes are described in Table 3. Response and survival outcome after re-irradiation was evaluated in 8 of all 9 patients. The patient (patient no. 5) who incompleted re-irradiation died 1.6 months after and the outcome could not be evaluated. Six patients (75\%) of the evaluated 8 patients showed response after reirradiation. Among them, 4 patients showed complete clinical

Table 2. Treatment characteristics

\begin{tabular}{cllcrlrrr}
\hline Patient no. & $\begin{array}{c}\text { Prior RT } \\
\text { setting }\end{array}$ & $\begin{array}{c}\text { Prior RT } \\
\text { technique }\end{array}$ & $\begin{array}{c}\text { Prior RT dose } \\
\left(\mathrm{Gy} \mathrm{y}_{10}\right)\end{array}$ & $\begin{array}{c}\mathrm{RT} \text { interval } \\
(\mathrm{mo})\end{array}$ & $\begin{array}{c}\text { Re-RT } \\
\text { setting }\end{array}$ & $\begin{array}{c}\text { Re-RT } \\
\text { PTV }\left(\mathrm{cm}^{3}\right)\end{array}$ & $\begin{array}{c}\text { Re-RT } \\
\text { dose }\left(\mathrm{Gy} y_{10}\right)\end{array}$ & $\begin{array}{c}\text { Total RT } \\
\text { dose }\left(\mathrm{Gy}{ }_{10}\right)\end{array}$ \\
\hline 1 & Postop RT & IMRT & 50.4 & 4 & RT alone & 179.33 & 47.5 & 97.9 \\
2 & Postop CCRT & 2D & 55.8 & 51 & CCRT & 133.85 & 36.0 & 91.8 \\
3 & Def CCRT & 3D & 73.8 & 25 & Sequential CRT & 14.84 & 46.7 & 120.5 \\
4 & Def CCRT & 3D & 72.0 & 4 & RT alone & 51.72 & 56.9 & 128.9 \\
5 & Postop CCRT & 3D & 67.1 & 5 & RT alone & 309.24 & 38.7 & 105.8 \\
6 & Postop RT & 2D & 59.4 & 117 & CCRT & 340.09 & 56.9 & 116.3 \\
7 & Def CCRT & 3D & 73.8 & 15 & RT alone & 14.58 & 50.8 & 124.6 \\
8 & Postop RT & 3D & 61.0 & 30 & CCRT & 107.71 & 67.1 & 128.1 \\
9 & Postop CCRT & 2D & 64.8 & 137 & RT alone & 98.81 & 40.7 & 105.5 \\
\hline
\end{tabular}

RT, radiotherapy; Re-RT, re-irradiation; PTV, planning target volume; Postop, postoperative; CCRT, concurrent chemoradiation; Def, definitive; IMRT, intensity-modulated radiotherapy; CRT, chemoradiation; 2D, 2-dimensional; 3D, 3-dimensional.

Table 3. Treatment outcomes

\begin{tabular}{|c|c|c|c|c|c|c|}
\hline Patient no. & Tx response & LRRFS (mo) & OS (mo) & Failure site & Survival status & Cause of death \\
\hline 1 & SD & 3.4 & 4.1 & Local & Death & Cancer \\
\hline 2 & SD & 7.4 & 28.1 & Local & Death & Cancer \\
\hline 3 & $\mathrm{CR}$ & 11.9 & 11.9 & Distant & Death & Cancer \\
\hline 4 & $\mathrm{CR}$ & 75.1 & 75.1 & No relapse & Alive & - \\
\hline 5 & - & - & 2.5 & - & Death & Cancer \\
\hline 6 & PR & 9.4 & 10.7 & Local + distant & Death & Cancer \\
\hline 7 & $\mathrm{CR}$ & 20.3 & 20.3 & No relapse & Death & Non-cancer \\
\hline 8 & $C R$ & 22.2 & 48.0 & Local + distant & Alive & - \\
\hline 9 & PR & 43.8 & 43.8 & No relapse & Alive & - \\
\hline
\end{tabular}

TX, treatment; LRRFS, locoregional relapse-free survival; OS, overall survival; SD, stable disease; CR, complete response; PR, partial response. 

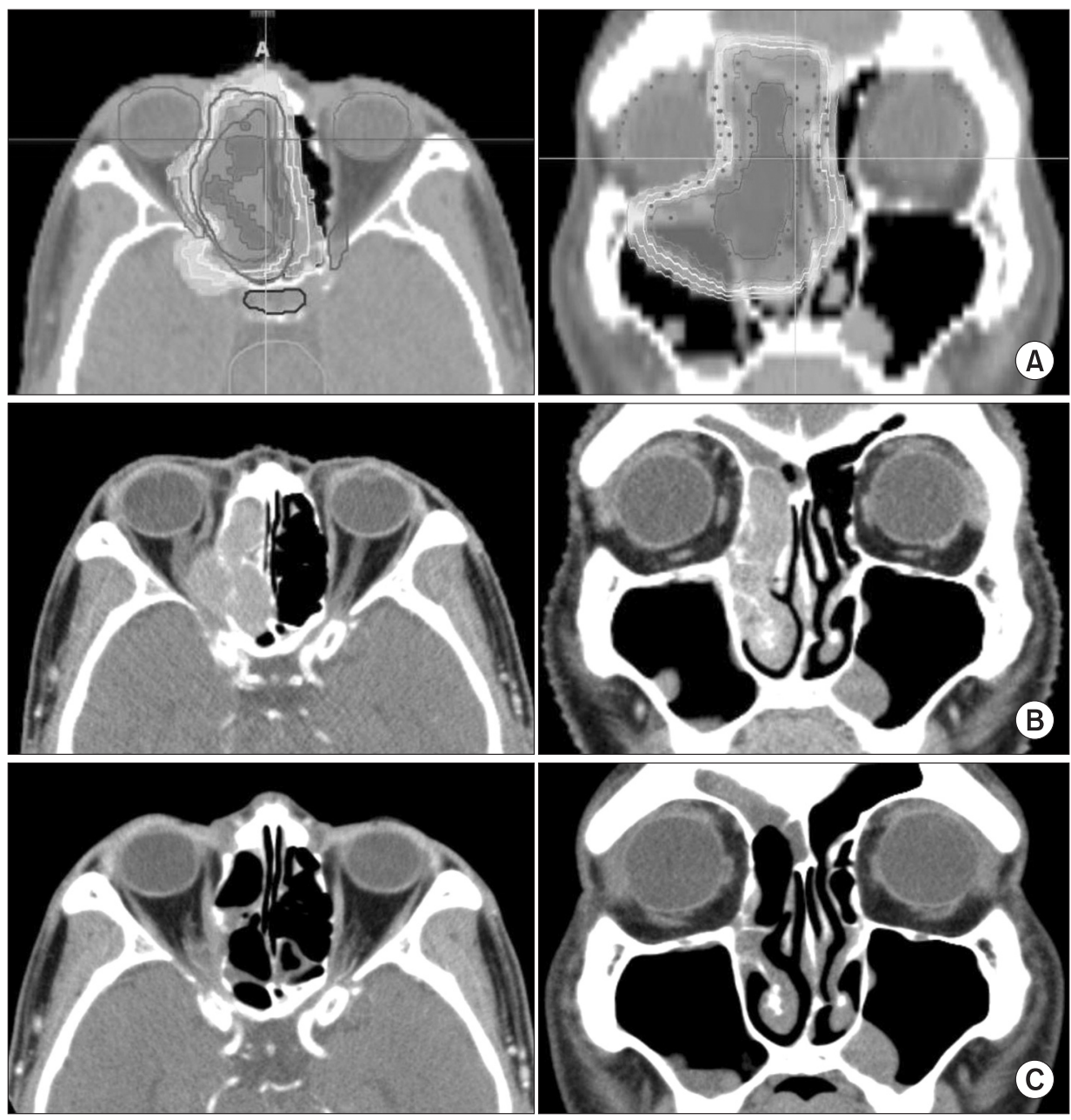

Fig. 1. Planning image and computed tomography (CT) image follow-up as representative example. (A) Reirradiation dose distribution image (axial and coronal). Axial and coronal images of CT paranasal sinuses (B) before re-irradiation and (C) after reirradiation.

and radiographic response while 2 had partial response. The remaining 2 patients had stable disease after re-irradiation. Fig. 1 is representative of a case in which complete response (CR) was achieved after re-irradiation.

The median LRRFS after re-irradiation of the 8 patients was 11.9 months (range, 3.4 to 75.1 months) and 5 patients eventually developed relapse by the time of analysis. Two patients had in-field relapses, 1 patient had out-field relapse, and 2 patients had simultaneous in- and out-field relapses. First sites of distant metastases were axilla and lung. One-year LRRFS rate was 50\% and 2-year LRRFS rate was 37.5\%.

At the start of re-irradiation, 7 of all 9 patients had symptoms related to recurrent lesions. Among them, 6 patients (86\%) experienced improved symptom during or after reirradiation-pain relief in 2 of 3 patients, improved swallowing discomfort in 2 patients, cessation of hemoptysis in 1 patient, and alleviated diplopia in 1 patient.

The median OS of the 8 patients who completed the planned re-irradiation schedules were 20.3 months (range, 4.1 to 75.1

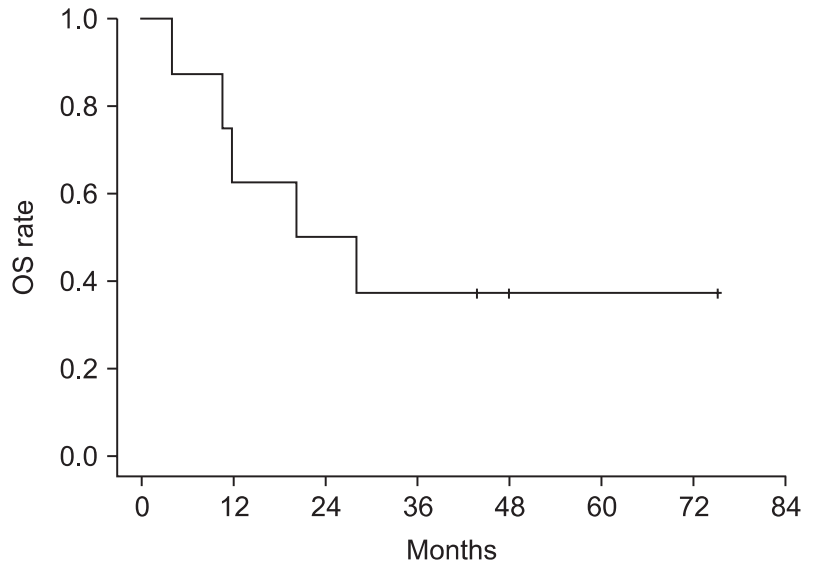

Fig. 2. Kaplan-Meier overall survival (OS) curves.

months). One-year OS rate was $62.5 \%$ and 2-year OS rate was $50 \%$ (Fig. 2). At the time of analysis, 3 of the total 9 patients were alive and 2 of the 3 patients were at disease-free state. The median OS of these alive patients was 48 months (range, 
Table 4. Late toxicities of prior RT and acute/late toxicities of Re-RT

\begin{tabular}{cll}
\hline Patient no. & Late toxicities of prior RT & \multicolumn{1}{c}{ Acute $^{\text {a) } / \text { late }}{ }^{\text {b) }}$ toxicities of Re-RT } \\
\hline 1 & Grade 2 xerostomia & Grade 2 mucositis \\
2 & - & Grade 2 dysphagia, Grade 1 hemoptysis \\
3 & Grade 1 hypothyroidism & Grade 2 mucositis \\
5 & Grade 2 xerostomia & Grade 1 eyelid swelling \\
6 & Grade 2 xerostomia & Grade 2 mucositis \\
7 & - & Grade 2 dysphagia \\
8 & - & Grade 2 dysphagia, Grade 1 hemoptysis \\
9 & Grade 1 otitis media & Grade 3 leukopenia, Grade 2 dysphagia \\
\end{tabular}

$\mathrm{RT}$, radiotherapy; $\mathrm{Re}-\mathrm{RT}$, re-irradiation.

${ }^{\text {a) }}$ Acute toxicities except dermatitis, ${ }^{\text {b) }}$ late toxicities.

43.8 to 75.1 months). Five cancer-related deaths by disease progression were observed, and one patient died from noncancer medical problem. Patient no. 7 achieved complete response after the second course of radiation, but she died 19.1 months after the end of RT from dementia which was diagnosed before the HNC treatment.

Patients with recurrence and yet a long-term survival of over 2 years were patient no. 2 and 8. Patient no. 2 was given 6 cycles of docetaxel-cisplatin chemotherapy after relapse but the disease progressed anyhow and no further chemotherapy was given because the patient refused. This patient expired due to dyspnea caused by local progression. The other patient, patient no. 8, refused further treatment after the diagnosis of recurrence and is currently still alive to date with disease progression.

\section{Toxicity}

Before the re-irradiation, treatment toxicity related to prior RT was recorded to evaluate adverse effects of re-irradiation separately. Treatment toxicity details were described in Table 4. Because of the prior RT, 3 patients already had grade 2 xerostomia, and each of the patients had grade 1 trismus, otitis media, and hypothyroidism, respectively. Acute toxicity, which was observed during or within 1 to 3 months after the completion of re-irradiation, was limited only to grade 1 or 2 toxicities except in one patient with grade 3 leukopenia. This was in patient no. 8 who underwent concurrent chemoradiotherapy. Four patients with grade 2 dysphagia, 3 patients with grade 2 oral mucositis, 2 patients with grade 1 hemoptysis, and 1 patient with grade 1 eyelid swelling were observed. All patients experienced grade 1 or 2 dermatitis as well. Late toxicity was observed in only one patient by the last follow-up. Patient no. 9 complained of grade 2 trismus and grade 2 osteonecrosis.

\section{Discussion and Conclusion}

Re-irradiation to locoregionally recurrent unresectable head and neck tumors obtained better median survival than using palliative chemotherapy. However, re-irradiation has historically been considered highly dangerous due to high risk of complications and treatment-related morbidities reported from early HNC studies. Several studies using different fractionation schemes to increase tumor response and decrease normal tissue toxicity were performed thereafter, but in most studies, toxicities were still substantial. RTOG conducted a phase II study (RTOG 96-10) in unresectable recurrent HNC with an altered fractionated regimen [10]. The study incorporated concurrent 5-fluorouracil, hydroxyurea chemotherapy, and radiation up to 60 Gy in 1.5 Gy per fraction, twice a day schedule. The 2- and 5-year OS was $15.2 \%$ and $3.8 \%$. The observed grade 5 acute toxicity was $7.6 \%$ and grade 4 acute toxicity was $17.7 \%$. Grade 4 late toxicities were found in 3.0\%. In another study, De Crevoisier et al. [11] compared three different radiation therapy schedules (2 Gy per day, total 65 Gy; 2 Gy per day, total 60 Gy; 1.5 Gy per fraction twice a day, total $60 \mathrm{~Gy}$ ). The $0 \mathrm{~S}$ rate at 2 and 5 years were $21 \%$ and $9 \%$, but grade 4 mucositis was detected in $14 \%$ of cases and late complications were also substantial (cervical fibrosis 41\%, mucosal necrosis 21\%, osteoradionecrosis $8 \%$, and trismus 30\%) with 5 patient dying of carotid hemorrhage. In multivariate analysis, re-irradiated volume was revealed as the only factor associated with risk of mortality.

With the improvement of radiation technique, IMRT was beginning to be applied in the field. It could generate better conformality decreasing irradiated volume to normal organs and increasing target dose delivery. Because most of the previously irradiated HNC recurred near already irradiated 
sites, improvement of locoregional therapeutic ratio was regarded as the key of cure. With IMRT, the curative chances were increased as well as the treatment toxicity was decreased. Lee et al. [13] reported experience of Memorial Sloan-Kettering Cancer Center. In the study, IMRT re-irradiation achieved a 2-year locoregional control of 52\% compared to 20\% of nonIMRT, and patients with higher locoregional control rates had better OS (1-year OS 56\% and 2-year OS 37\%). Acute toxicities of grade 3 or more were $23 \%$ and only $4 \%$ of grade 4 late toxicities was observed. Despite the high cumulative doses of radiation, no grade 5 toxicity was observed. Another IMRT trial [14] reported also favored in use of IMRT with outcomes of 22.8 months for mean estimated survival and acceptable complications in unresectable re-irradiation patients.

Furthermore, in head and neck area, the distance between critical organs and target volumes may be very close in many cases. For that reason, overcoming potential uncertainties in patient set up and intrafractional/interfractional motion during the RT course is especially very important compared to other sites. However, Chen et al. [17] reported that daily interfractional motion shifts of the head and neck area were significant even with thermoplastic mask, $>5 \mathrm{~mm}$ in $8 \%$ to $19 \%$. The result suggested that minimizing these uncertainties using IGRT complemented to IMRT is very useful in improving the therapeutic ratio of head and neck irradiation. Den et al. [15] and Wang et al. [16] reported that mean interfractional shift was decreased to 1.1 to $1.8 \mathrm{~mm}$ using IG-IMRT. The study on IG-IMRT re-irradiation to the recurrent HNC [17] showed the supported findings with improved outcomes. They reported improved 2-year outcomes with 65\% in-field control and $\mathrm{OS}$ rates of $40 \%$. There were no complications which needed treatment-related hospitalization. Helical Tomotherapy was given with daily MVCT performed and daily CT image fused with planning CT to minimize set up error. In addition, checking the fused image by physician was also performed each day in our institution. These everyday procedures may have contributed in reducing irradiation volume of normal organ and not missing target.

Fewer complications and improved survival outcomes with IG-IMRT were observed as well in our institution. The 1and 2-year OS rates were $62.5 \%$ and $50.0 \%$, respectively. Locoregional control rate achieved by re-irradiation was $75 \%$. Only 1 patient appeared with grade 3 acute toxicity and 1 patient with grade 2 late toxicity. Due to the retrospective nature of our series and two-thirds of patients being dead at the time of analysis, assessment of late toxicities is quite difficult. However, outcomes of 3 alive patients with the median follow-up 49 months showed no severe late toxicity.

The optimal treatment volume is uncertain [18] but several studies reported that smaller irradiated volume resulted in better survival outcomes. Langlois et al. [19] reported that higher local control was obtained when the cumulative volume of irradiation was reduced. De Crevoisier et al. [11] reported that re-irradiated volume was the only factor significantly associated with the risk of death in multivariate analysis. In our study, the absolute median PTV of re-irradiation was relatively small with the value of $107.71 \mathrm{~cm}^{3}$ (range, 14.58 to $340.09 \mathrm{~cm}^{3}$ ). To minimize overlapped RT field and re-irradiation volume, no elective nodal irradiation was performed except for the cases in which adjacent nodal matastases had been detected. Michigan University reported that most of the recurrences were detected near GTV [20] and that lymphatic draining pattern could be altered after prior RT. These resulted in unexpected lymph node metastases not matching standard lymphatic drainage in re-irradiated HNC. Therefore, in our institution, elective irradiation of uninvolved nodal regions was performed only in three patients (patient no. 1, 5, and 6) in our institution.

Because the PTV dose of re-irradiation was determined by cumulative dose of normal organs, patients with larger PTVs have possibility of being prescribed lower re-irradiation doses than others. Therefore, RT dose conjunction with PTV could largely contribute in differing outcomes. In our study, patients who obtained CR after re-irradiation (patient no. 3, 4, 7, and 8) were delivered median cumulative RT dose of $125.9 \mathrm{~Gy} y_{10}$ with a range from 120.5 to $128.9 \mathrm{~Gy}_{10}$. It is supported by the outcome of Sulman et al. [21]. They reported that cumulative RT dose over 119.4 Gy was associated with improved locoregional control in multivariate analysis. Several studies reported another prognostic factor to improve local control as a reirradiation dose of more than 50 to $60 \mathrm{~Gy}[22,23]$. However, in our study, several patients have relatively poor initial RT characteristics and we delivered relatively safe re-irradiation dose to the PTV. The median re-irradiation dose was $47.5 \mathrm{~Gy}_{10}$ and only one patient (patient no. 8) was prescribed over $60 \mathrm{~Gy}$ $\left(67.1 \mathrm{~Gy}_{10}\right)$. It could have been conducive to somewhat shorter period of local control than former studies despite of our other good outcomes. In addition, among our patients, a third of the patients (patient no. 2, 6, and 9) had priorly been given 2D RT. By using 2D RT, the irradiated volume of normal tissues or OARs were generally larger than that of $3 \mathrm{D}$ conformal RT. The re-irradiation dose was determined by the cumulative 
dose of OARs, therefore relatively low re-irradiation dose was considered in those patients. Among 3 patients who have been delivered a re-irradiation dose under $45 \mathrm{~Gy}_{10}, 2$ patients had been treated with previous 2D RT (patients no. 2 and 9). Another patient is patient no. 5, who incompleted RT. Patient no. 6 was also priorly treated with 2D RT but the prescribed reirradiation dose was $58.3 \mathrm{~Gy}_{10}$ owing to the quite long interval between the two courses of RT (117 months) and to the fact that the critical organs were located relatively far away from the recurrent lesion. Short RT interval was another factor that disturbed prescribing sufficient re-irradiation dose for tumor control. Spencer et al. [10] and Duprez et al. [24] reported longer survival of patients who had longer RT interval. In our study, over $30 \%$ of patients had only 4 or 5 months of RT interval (patient no. 1, 4, and 5) and were prescribed relatively low doses except for patient no. 4 . This patient could be prescribed with a re-irradiation dose of up to $56.9 \mathrm{~Gy}_{10}$ because the neural OARs, optic nerves, and chiasm, were not highly irradiated in prior RT.

In literature, suggested important prognostic factors of outcomes in recurrent HNC were time interval between the two RT courses, irradiation volume, re-irradiation dose, predisposing organ dysfunction, and comorbidities. However, we could not analyze the factors statistically because of the small patient number. Furthermore, the heterogeneity of disease characteristics in our study could be regarded as insufficient for reporting the outcomes thoroughly enough. However, these ranges of heterogeneity were seen in almost any other published retrospective studies and the outcomes varied widely, from $25 \%$ to $85 \%$. The results of our retrospective study are meaningful in that they are relatively encouraging compared to historical data, despite having many elements generally reported as poor prognostic factors. Although 8 of the 9 patients' cancer histology were squamous cell carcinoma and all of them locoregionally advanced and inoperably recurred, our data showed comparably long survival and low toxicities. It indicates the efficacy and safety of reirradiation using IG-IMRT.

In addition, the symptoms caused by recurrent lesions, were improved in 6 of 7 patients (87\%) after re-irradiation. These results suggest that IG-IMRT re-irradiation could be clinically important treatment option in palliative locoregional disease control as well as survival improvement for recurrent HNC patients.

Nowadays, most of the radiotherapies of primary HNC use 3D conformal RT or IG-/IMRT and the use of 2D RT is reduced. The development of treatment in terms of surgery, chemotherapy as well as RT has increased local control rate and progressionfree survival of primary HNC. This may have contributed in reduction of irradiated volume and the increase of $\mathrm{RT}$ interval and utilization of higher dose delivery of re-irradiation in unresectable recurrent HNC.

Furthermore, the efforts to improve outcomes of reirradiation have been performed in various ways to date. Stereotactic body radiotherapy (SBRT) for previously irradiated recurrent HNC has been studied recently to escalate the dose on the recurrent tumor while sparing OARs and non-tumor soft tissues. SBRT consists of image-guided delivery of 1 to 10 fractions with relatively large fraction size. The utility of SBRT is thought to have overcome the radio-resistance of tumor because of the fundamentally different biologic effect compared to conventional RT. However, the outcomes of SBRT have been variable. Rwigema et al. [25] reported that higher SBRT doses, 40 to $50 \mathrm{~Gy}$, were associated with improved locoregional control with no late grade 4-5 toxicities. On the other hand, Cengiz et al. [26] reported high rate of carotid artery rupture (17\%) with a median dose of 30 Gy. Applying SBRT may be more tedious in compensating RT dose for adjacent OARs compared to fractionated RT if the tumor is bulky or very close to normal organs with ill-defined tumor margin. Further study is required to select appropriate patients who may benefit from SBRT.

Other clinical researches using targeted agent in conjunction with re-irradiation to enhance therapeutic effect of recurrent HNC by increasing specificity have also been performed recently. Currently the most focused targeted agent is epidermal growth factor receptor inhibitor, such as cetuximab, which has shown clinical benefit in primary HNC $[27,28]$. Other novel target agents, such as small molecule inhibitor (erlotinib) or proteasome inhibitor (bortezomib) are currently being investigated $[29,30]$. These targeted therapies with reirradiation is thought to be relatively tolerable but no definitive conclusions has been derived yet and further study is required in this area as well.

There is no randomized trial data of re-irradiation for previously irradiated recurrent HNC to date. And comparisons of outcomes among heterogeneous retrospective studies are limited. Therefore the decision-making in selecting treatment option of re-irradiation for recurrent HNC and in the process of radiation treatment planning needs to consider these several prognostic factors and former studies carefully with the principles to optimize outcomes. In our institution, the 
decision-making in selecting treatment option of Tomotherapy re-irradiation for recurrent $\mathrm{HNC}$ and radiation treatment planning was performed by the same radiation oncologist with uniform treatment strategy. This may have contributed in minimizing differences by physician's principles in treatment plans and processes between cases.

In conclusion, re-irradiation using Helical Tomotherapy as IG-IMRT for previously irradiated patients with unresectable locoregionally recurrent HNC may be an effective treatment option with long-term survival and acceptable toxicities. Further clinical studies with larger patient numbers and better homogeneity of patient population in properly selecting reirradiation patients are needed for better outcomes.

\section{Conflict of Interest}

No potential conflict of interest relevant to this article was reported.

\section{References}

1. Forastiere AA, Goepfert $H$, Maor M, et al. Concurrent chemotherapy and radiotherapy for organ preservation in advanced laryngeal cancer. N Engl J Med 2003;349:2091-8.

2. Overgaard J, Hansen HS, Specht $L$, et al. Five compared with six fractions per week of conventional radiotherapy of squamous-cell carcinoma of head and neck: DAHANCA 6 and 7 randomised controlled trial. Lancet 2003;362:933-40.

3. Bernier J, Domenge C, Ozsahin M, et al. Postoperative irradiation with or without concomitant chemotherapy for locally advanced head and neck cancer. N Engl J Med 2004;350:1945-52.

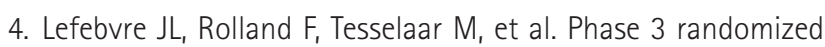
trial on larynx preservation comparing sequential vs alternating chemotherapy and radiotherapy. J Natl Cancer Inst 2009;101:142-52.

5. Spector JG, Sessions DG, Haughey BH, et al. Delayed regional metastases, distant metastases, and second primary malignancies in squamous cell carcinomas of the larynx and hypopharynx. Laryngoscope 2001;111:1079-87.

6. Wong SJ, Machtay M, Li Y. Locally recurrent, previously irradiated head and neck cancer: concurrent re-irradiation and chemotherapy, or chemotherapy alone? J Clin Oncol 2006;24:2653-8.

7. Jacobs C, Lyman G, Velez-Garcia E, et al. A phase III randomized study comparing cisplatin and fluorouracil as single agents and in combination for advanced squamous cell carcinoma of the head and neck. J Clin Oncol 1992;10:257-63.
8. Forastiere AA, Neuberg D, Taylor SG 4th, DeConti R, Adams G. Phase II evaluation of Taxol in advanced head and neck cancer: an Eastern Cooperative Oncology group trial. J Nat Cancer Inst Monogr 1993;(15):181-4.

9. Kasperts N, Slotman B, Leemans CR, Langendijk JA. A review on re-irradiation for recurrent and second primary head and neck cancer. Oral Oncol 2005;41:225-43.

10. Spencer SA, Harris J, Wheeler RH, et al. Final report of RTOG 9610, a multi-institutional trial of reirradiation and chemotherapy for unresectable recurrent squamous cell carcinoma of the head and neck. Head Neck 2008;30:281-8.

11. De Crevoisier R, Bourhis J, Domenge $C$, et al. Full-dose reirradiation for unresectable head and neck carcinoma: experience at the Gustave-Roussy Institute in a series of 169 patients. J Clin Oncol 1998;16:3556-62.

12. Langer CJ, Harris J, Horwitz EM, et al. Phase II study of lowdose paclitaxel and cisplatin in combination with splitcourse concomitant twice-daily reirradiation in recurrent squamous cell carcinoma of the head and neck: results of Radiation Therapy Oncology Group Protocol 9911. J Clin Oncol 2007;25:4800-5.

13. Lee N, Chan K, Bekelman JE, et al. Salvage re-irradiation for recurrent head and neck cancer. Int J Radiat Oncol Biol Phys 2007;68:731-40.

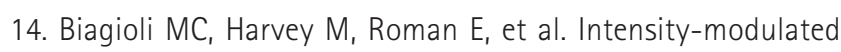
radiotherapy with concurrent chemotherapy for previously irradiated, recurrent head and neck cancer. Int J Radiat Oncol Biol Phys 2007;69:1067-73.

15. Den RB, Doemer A, Kubicek G, et al. Daily image guidance with cone-beam computed tomography for head-and-neck cancer intensity-modulated radiotherapy: a prospective study. Int J Radiat Oncol Biol Phys 2010;76:1353-9.

16. Wang J, Bai $S$, Chen $N$, et al. The clinical feasibility and effect of online cone beam computer tomography-guided intensitymodulated radiotherapy for nasopharyngeal cancer. Radiother Oncol 2009;90:221-7.

17. Chen AM, Farwell DG, Luu Q, Cheng S, Donald PJ, Purdy JA. Prospective trial of high-dose reirradiation using daily image guidance with intensity-modulated radiotherapy for recurrent and second primary head-and-neck cancer. Int J Radiat Oncol Biol Phys 2011;80:669-76.

18. McDonald MW, Lawson J, Garg MK, et al. ACR Appropriateness Criteria retreatment of recurrent head and neck cancer after prior definitive radiation expert panel on radiation oncology-head and neck cancer. Int J Radiat Oncol Biol Phys 2011;80:1292-8.

19. Langlois D, Eschwege F, Kramar A, Richard JM. Reirradiation of head and neck cancers: presentation of 35 cases treated at the Gustave Roussy Institute. Radiother Oncol 1985;3:27-33.

20. Popovtzer A, Gluck I, Chepeha DB, et al. The pattern of failure 
after reirradiation of recurrent squamous cell head and neck cancer: implications for defining the targets. Int J Radiat Oncol Biol Phys 2009;74:1342-7.

21. Sulman EP, Schwartz DL, Le $\Pi$, et al. IMRT reirradiation of head and neck cancer-disease control and morbidity outcomes. Int J Radiat Oncol Biol Phys 2009;73:399-409.

22. Stevens KR Jr, Britsch A, Moss WT. High-dose reirradiation of head and neck cancer with curative intent. Int J Radiat Oncol Biol Phys 1994;29:687-98.

23. Pomp J, Levendag PC, van Putten WL. Reirradiation of recurrent tumors in the head and neck. Am J Clin Oncol 1988;11:543-9.

24. Duprez F, Madani I, Bonte K, et al. Intensity-modulated radiotherapy for recurrent and second primary head and neck cancer in previously irradiated territory. Radiother Oncol 2009;93:563-9.

25. Rwigema JC, Heron DE, Ferris RL, et al. The impact of tumor volume and radiotherapy dose on outcome in previously irradiated recurrent squamous cell carcinoma of the head and neck treated with stereotactic body radiation therapy. Am J
Clin Oncol 2011;34:372-9.

26. Cengiz M, Ozyigit $G$, Yazici $G$, et al. Salvage reirradiaton with stereotactic body radiotherapy for locally recurrent head-andneck tumors. Int J Radiat Oncol Biol Phys 2011;81:104-9.

27. Balermpas $P$, Hambek $M$, Seitz 0 , Rodel $C$, Weiss $C$. Combined cetuximab and reirradiation for locoregional recurrent and inoperable squamous cell carcinoma of the head and neck. Strahlenther Onkol 2009;185:775-81.

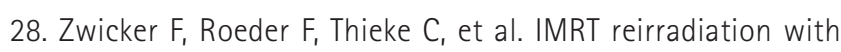
concurrent cetuximab immunotherapy in recurrent head and neck cancer. Strahlenther Onkol 2011;187:32-8.

29. Kao J, Genden EM, Chen CT, et al. Phase 1 trial of concurrent erlotinib, celecoxib, and reirradiation for recurrent head and neck cancer. Cancer 2011;117:3173-81.

30. Van Waes $C$, Chang AA, Lebowitz PF, et al. Inhibition of nuclear factor-kappaB and target genes during combined therapy with proteasome inhibitor bortezomib and reirradiation in patients with recurrent head-and-neck squamous cell carcinoma. Int J Radiat Oncol Biol Phys 2005;63:1400-12. 